\title{
BeeCheck - Plataforma para monitoramento de colmeias, colônias e apiários
}

\author{
Victor Kayan de A. Lima ${ }^{1}$, Sadrak Lyon D. Pontes ${ }^{1}$, Claudio R. de Medeiros ${ }^{1}$, \\ Gerson V. Marques ${ }^{1}$, Atonio Abreu da S. Neto ${ }^{1}$, Elenilson Vieira da S. Filho ${ }^{1}$ \\ ${ }^{1}$ Instituto Federal de Educação, Ciência e Tecnologia do Rio Grande do Norte (IFRN) \\ Código Postal 59900-000 - Pau dos Ferros, RN - Brasil \\ \{victor.k, lyon.pontes\}eescolar.ifrn.edu.br, \\ \{claudiorodrigozh, vianagerson2011\}@gmail.com, \\ \{elenilson.vieira, antonio.neto3\}eifrn.edu.br
}

\begin{abstract}
The bees' breeding is one of the most important agricultural activities in Brazil, it is from this work that comes honey that is prominent in the world. This status gives rise to concern about the production of honey and the need to monitor the development of hives. The lack of active and free systems dedicated to the area of beekeeping permeates the lives of beekeepers. Therefore, it is aimed to present the development of the platform called BeeCheck, where users can manage all the information of their apiaries, thus providing a greater convenience and security in the storage of this data. For the development, were used free tools and with great acceptance in the market, aiming a practical construction and with low cost in the final product.
\end{abstract}

Resumo. A apicultura é uma das mais importantes atividades agropecuárias no Brasil. É desse trabalho que vem o mel que é destaque no mundo. Esse status gera preocupação com a produção do mel. Nesse sentido, a carência de sistemas ativos e gratuitos voltados para a área da apicultura permeiam a vida dos apicultores. Assim, é visado apresentar o desenvolvimento da plataforma BeeCheck, na qual os usuários poderão gerenciar todas as informações de seus apiários, proporcionando uma maior praticidade e segurança no armazenamento desses dados. Para o desenvolvimento, foram utilizadas ferramentas gratuitas e com bastante aceitação no mercado, a fim de ter uma construção prática e com baixo custo no produto final.

\section{Introdução}

A criação de abelhas é uma das mais importantes atividades agropecuárias no Brasil. É desse trabalho que vem a renda de diversas famílias. São esses insetos que fazem o mel, um produto natural que tem papel importante na nutrição humana. Ademais, é amplamente utilizado na medicina, como, por exemplo, em tratamentos terapêuticos [Dutra, 2016; Silva et al., 2008].

\subsection{Descrição do problema}

Segundo Oliveira (2017), o mel brasileiro destaca-se dentre os melhores do mundo em virtude de sua alta qualidade. Esse status mundial gera, por outro lado, preocupação com a sua produção e a necessidade de acompanhar o desenvolvimento das colônias. 
Nessa perspectiva, existem formas de rastreio que garantem ao consumidor um produto seguro e saudável, por meio do controle de todas as fases de produção. Atualmente, essa supervisão, na maioria das vezes, é realizada de forma manual por pequenos produtores. Tal trabalho é feito através de papel e caneta, o que torna o manejo dos dados mais dificultoso, além de ser um procedimento lento e inseguro em virtude de as informações serem armazenadas de forma rudimentar. Esses fatos citados podem comprometer o bom desempenho das abelhas e a possibilidade de lucros maiores por parte dos apicultores.

Dessa forma, tendo em vista a importância do mel e da sua qualidade, este trabalho visa apresentar uma solução denominada BeeCheck. Esse sistema computacional é composto por dois módulos distintos: o módulo do apicultor ou responsável por acompanhar os apiários (aplicativo móvel) e o módulo do técnico em apicultura (aplicação web); além de uma API para a comunicação dos dados. Sob esse prisma, o objetivo do projeto é auxiliar apicultores e técnicos em apicultura no processo de manejo apícola e Escrituração Zootécnica, através do armazenamento das informações sobre suas colônias, colmeias e apiários (com o aplicativo móvel); bem como analisar as referidas informações nos procedimentos realizados em cada visita às colmeias por meio da aplicação web.

\section{Referencial Teórico}

Para um bom desenvolvimento do trabalho apícola, faz-se necessário o armazenamento das informações sobre o estado das colmeias, das colônias e acerca do ambiente onde elas se localizam. Nesse sentido, nenhuma atividade econômica pode prosperar e se manter a longo prazo sem que se conheça o custo de seus produtos e os fatores que o determinam [Souza, 2007].

\subsection{A importância da Apicultura}

A apicultura, trabalho da criação de abelhas, é de suma importância para o desenvolvimento da economia e do meio ambiente brasileiros. Nesse contexto, o Brasil se destaca por ser, atualmente, o sexto maior produtor de mel do mundo [Bacaxixi et al., 2011] e esse status mundial tende a crescer.

\subsection{Manejo de Apiários e Escrituração Zootécnica}

A fim de aumentar a eficiência da produção bem como o resultado do mel e outros produtos apícolas, o apicultor - profissional responsável por criar as abelhas - precisa observar todos os fatores que envolvem o apiário.

Dessa forma, alguns agentes precisam guiar o desenvolvimento das abelhas, por exemplo: definir onde será posicionado o apiário; o período da flora apícola; a presença de locais abrigados por árvores; sombreamento; topografia; facilidade de acesos; segurança e limpeza do local e utensílios [A.B.E.L.H.A., 2019]. Portanto, a observação, estudo e manutenção desses fatores se consolida como manejo apícola.

Nesse contexto, de acordo com Lôbo (2007), a escrituração zootécnica é o conjunto de práticas que diz respeito a observação e anotação de atividades rurais realizadas, esse processo permite um controle total das condições e do desenvolvimento da atividade realizada com os animais.

\subsection{Trabalhos Relacionados}


Estudos utilizando o auxílio de ferramentas eletrônicas para obtenção de dados relacionados às abelhas e a apicultura estão sendo altamente discutidos e vêm ganhando destaque. Nesse sentido, buscou-se aportes em trabalhos que visam realizar o monitoramento de colmeias e apiários, através de soluções tecnológicas.

Iordache (2019), propôs um sistema intitulado Caderno do apicultor, um aplicativo que permite realizar ações como: adicionar tarefas, registrar apiários, colmeias, colheitas, deslocamentos e gerenciar os cuidados veterinários.

O referido trabalho, assim como a plataforma BeeCheck, empregam o uso de um aplicativo móvel para registros de apiários e suas respectivas colmeias. Em relação às diferenças, destaca-se a forma com que os dados são utilizados; o BeeCheck dispõe de uma API para comunicação e armazenamento dos dados, enquanto na ferramenta de Iordache (2019) o armazenamento é local, dificultando a escalabilidade e compartilhamento dos dados.

\section{Arquitetura de Comunicação}

A arquitetura de software representa os componentes envolvidos no sistema, visando demonstrar o funcionamento, os objetivos, as propriedades externas e envolvimentos com outros softwares ou ferramentas. Nesse caso, a flexibilidade desejada para o sistema demanda que as aplicações implementem processos de negócio pertinente aos mesmos, de forma isolada, e que esses dados sejam compartilhados entre os módulos (web e móvel).

Sob o referido viés, a abordagem tradicional para a solução desse problema seria através de um Web Service que integra os sistemas através de mensagens no formato SOAP (Simple Object Access Protocol). Contudo, consoante Rodrigues (2009), "muitas vezes o desempenho do sistema é comprometido, devido ao tamanho das mensagens envelopadas no protocolo SOAP".

Portanto, dentre outras soluções, optou-se por uma arquitetura baseada no paradigma REST (Representational State Transfer). Nesse caso, os processos são compartilhados através de uma API, de modo mais satisfatório que o SOAP. Isso tem como objetivo reduzir o tráfego de dados, tendo em vista as dificuldades de conexão no campo, onde o aplicativo será utilizado.

Diante disso, os módulos, localizados em dispositivos distintos, trocam informações através de uma infraestrutura de rede. A aplicação móvel, a web e a API comunicam-se apenas passando mensagens; fatos esses que caracterizam a plataforma como um sistema distribuído [Coulouris, Dollimore e Kindberg, 2007].

\subsection{Fluxo de comunicação}

Aplicando esses pontos no caso do BeeCheck, em seu fluxo de uso normal, inicialmente o apicultor será o responsável por ir, de fato, até o apiário e coletar os dados, os quais serão inseridos no aplicativo móvel, através do preenchimento dos formulários referentes às visitas. Essas informações precisarão ser armazenadas remotamente; contudo, o aplicativo não possui acesso direto ao banco de dados. Portanto, a aplicação, utilizandose de requisições HTTP e seus verbos, repassa os dados através da API, a qual consegue acessar o banco e, nesse caso, persistir as informações.

Assim, uma vez que os dados se encontram armazenados remotamente, poderão ser acessados em ambas aplicações por intermédio da API, isto é, as informações tornam- 
se sincronizadas. Então, a API é responsável por realizar a comunicação, indiretamente, entre módulos, pois, de fato, interliga-os à base de dados. A Figura 1 traz um esquema gráfico do fluxo comunicativo e arquitetura da plataforma.

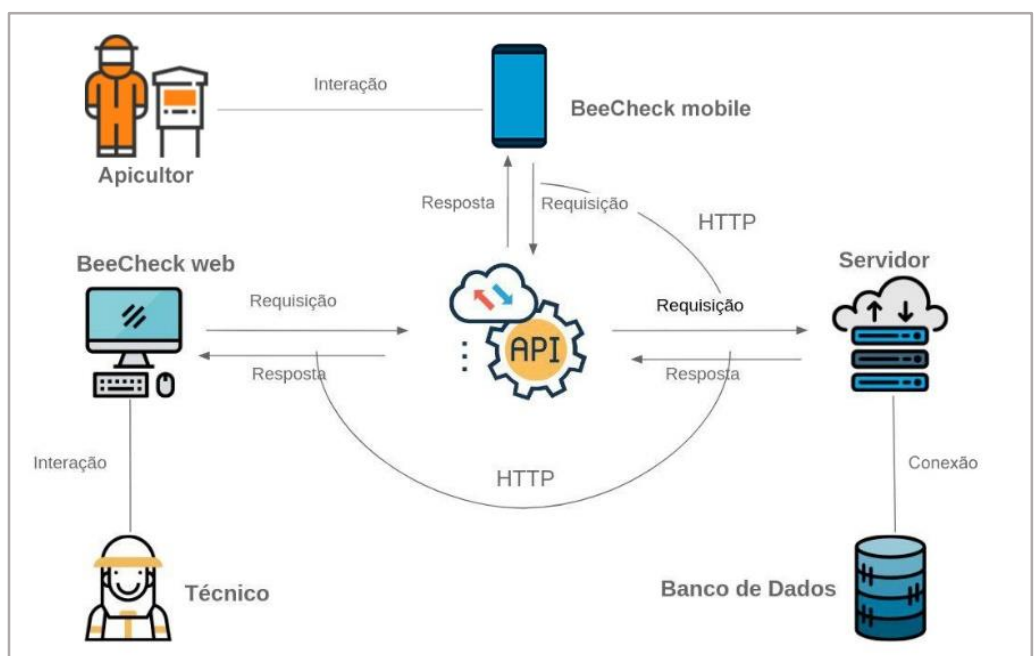

Figura 1. Arquitetura de comunicação do BeeCheck

Nesse cenário, para que as informações cadastradas no banco possam voltar e serem efetivamente utilizadas pelos módulos web e móvel, as aplicações devem realizar requisições a API. A nível de exemplificação, o fluxo, simplificado, do processo de realização de uma visita e visualização desta pelo sistema web é dado, respectivamente, por:

- entrada de dados pelo aplicativo;

- requisição HTTP: POST a API partindo do aplicativo;

- registro das informações na base de dados;

- requisição HTTP: GET a API partindo do website;

- recuperação das informações na base de dados;

- resposta da API ao website com os dados solicitados.

\section{BeeCheck Móvel}

Como supracitado, será função do apicultor utilizar o aplicativo para coletar os dados referentes a produção apícola. Para isso, dentre as funcionalidades disponíveis, estão: gerenciamento de colmeias e colônias, visitas e intervenções. Dessa forma, ao abrir o aplicativo, é exibida a primeira tela da Figura 2 abaixo. Os principais recursos contidos nessa tela são os informativos com os números dos apiários e colmeias cadastrados, bem como intervenções a seres feitas. Ademais, essa mesma tela mostra um mapa com a posição geográfica de cada apiário referente ao apicultor logado.

Para serem feitas intervenções, as informações registradas no aplicativo durante a visita percorrem todo o fluxo de comunicação descrito anteriormente até chegar no sistema web, onde o especialista em apicultura poderá visualizar os dados e dar sua reposta com o que deve ser feito. Dessa forma, de acordo com as informações cadastradas, o técnico responde com suas intervenções. Por exemplo: se um dado coletado durante a visita é "não há água perto da colmeia", algumas possíveis intervenções a serem feitas seriam "desloque a colmeia para um local que tenha água" ou "coloque água perto da colmeia". 

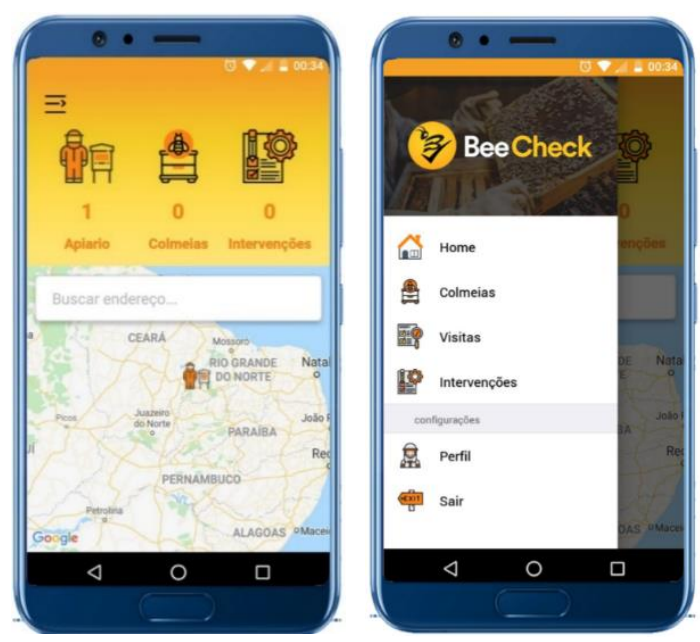

Figura 2. Tela inicial e barra de menu do aplicativo

\section{BeeCheck Web}

A utilização do sistema web será de responsabilidade dos técnicos em apicultura. Através dele, será possível: gerenciar apicultores, gerenciar apiários, visualizar visitas, realizar intervenções e gerar relatórios. Porém, para fazer o gerenciamento dos objetos supracitados, é necessário que o técnico efetue login no sistema web.

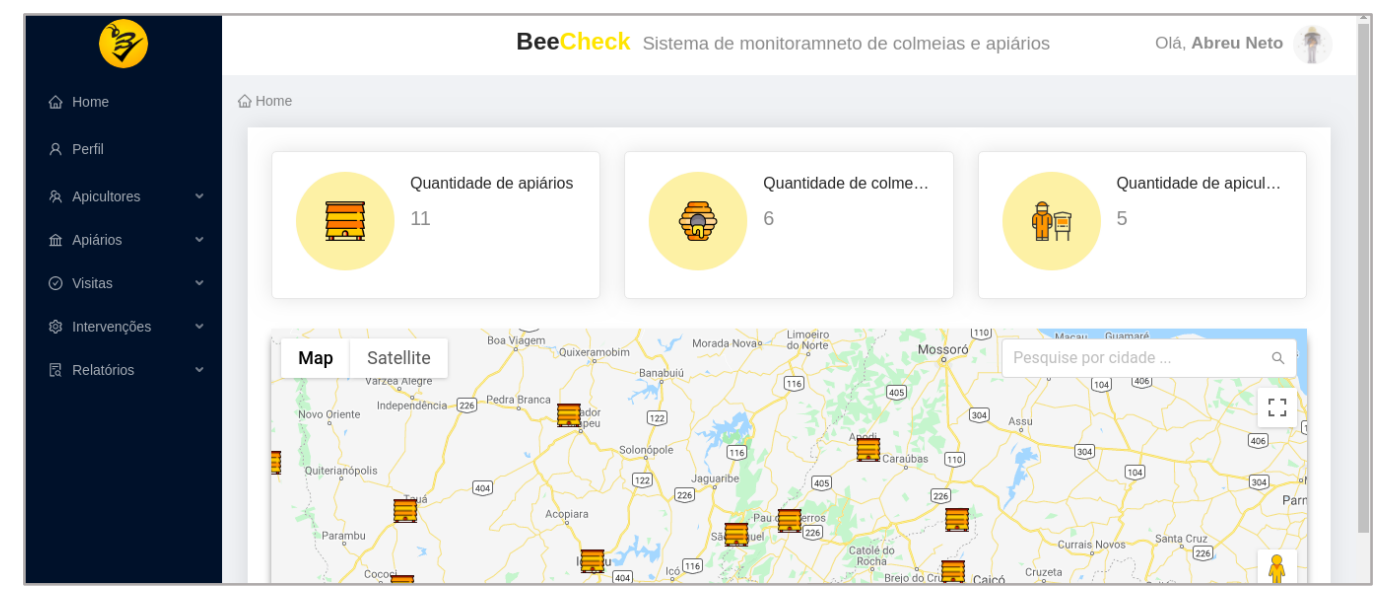

Figura 3. Tela inicial da aplicação web

A Figura 3 acima apresenta a tela inicial do sistema para um usuário devidamente autenticado. Nela são contidas informações como: quantidade de apiários, quantidade de colmeias em todos os apiários e a quantidade de apicultores que o técnico é responsável por gerenciar.

\section{Experimentos Realizados}

Para a construção do sistema, bem como análise das funcionalidades existentes e suas adequações com o objetivo proposto, foram realizadas visitas ao apiário do IFRN Campus Pau dos Ferros. Desse modo, foi possível vivenciar mais de perto a realidade do apicultor e simular casos de usos concretos. Sendo assim, foram percebidas, efetivamente, as dificuldades enfrentadas, permitindo uma esquematização mais realista e acessível das formas automatizadas de se fazer a Escrituração Zootécnica.

Sob o intuito de testar o aplicativo móvel, analisou-se os seguintes requisitos funcionais: cadastro de colmeia em um apiário, realização de visita e aplicação de intervenção. Para que o sistema funcione corretamente, é necessário conexão com a internet, tendo em vista que a API, utilizada em praticamente todos os processos, funciona 
a partir de comunicações HTTP. Portanto, diante do fato de que o apiário do IFRN Campus Pau dos Ferros (bem como a maioria dos apiários) não possui rede de área local sem fio, durante todos os testes foi utilizada a rede de dados da operadora de serviço telefônico, sendo a única opção de conexão viável para a situação.

Tendo isso em mente, realizou-se testes do tempo de duração de uma requisição a API em três cenários distintos de conexão com a internet, a fim de comparar uma situação de conectividade ideal com o caso real dentro do apiário, como mostra a Tabela 1. Desse modo, optou-se por testar os processos de cadastro de colmeia com foto (POST) e recuperação das intervenções dos apiários (GET). Para isso, com o fito de ser o mais realista possível, as requisições foram feitas através aplicativo móvel, por meio de um smartphone Samsung Galaxy M20. Nesse contexto, com o propósito de calcular o tempo médio e o desvio padrão, realizou-se 10 medições para cada tipo de requisição e cenário. Os dados das tabelas abaixo mostram a descrição dos locais onde foram realizados os testes bem como a qualidade da das conexões nesses locais:

Tabela 1. Latência, velocidades de download e upload nos cenários de uso da plataforma

\begin{tabular}{|c|c|c|c|}
\hline & $\begin{array}{c}\text { Latência } \\
\text { (média em ms) }\end{array}$ & $\begin{array}{c}\text { Download (velocidade } \\
\text { média em Mbps) }\end{array}$ & $\begin{array}{c}\text { Upload (velocidade } \\
\text { média em Mbps) }\end{array}$ \\
\hline Wi-Fi & 8,6 & 38,6 & 23,2 \\
\hline 4G na cidade & 126,8 & 12,28 & 7,83 \\
\hline 4G no apiário & 647,8 & 3,23 & 0,27 \\
\hline
\end{tabular}

Tabela 2. Teste de tempo de duração entre requisições e respostas da API

\begin{tabular}{|c|c|c|c|}
\hline & Wi-Fi & 4G na cidade & 4G no apiário \\
\hline Média do GET (ms) & 280 & 534,1 & 2508,5 \\
\hline Média do POST (ms) & 1690,1 & 2114,9 & 30293 \\
\hline Desvio padrão do GET & 30,74 & 357,97 & 5343,04 \\
\hline Desvio padrão do POST & 70,7 & 322,44 & 43464,04 \\
\hline
\end{tabular}

Para compor a Tabela 2 acima, foram calculados a média e o desvio padrão de cada conjunto de dados. A média sintetiza o tempo de resposta para cada tipo de requisição, e o desvio padrão aponta a variação entre as medições do conjunto. Logo, é válido destacar os valores do POST no Wi-Fi e no apiário: enquanto o tempo médio de resposta no primeiro cenário foi cerca de $1690 \mathrm{~ms}$, no local de criação das abelhas, com a conexão $4 \mathrm{G}$, foi de $30293 \mathrm{~ms}$, mostrando uma grande lentidão neste último. Ademais, nota-se que, no Wi-Fi, tem-se o desvio padrão do POST com um valor de $70,7 \mathrm{~ms}$; no apiário, o valor é de $43464,04 \mathrm{~ms}$, denunciando uma instabilidade exorbitantemente maior em relação aos outros cenários.

Nesse âmbito, dentro do apiário, ações que demandam um fluxo pequeno de dados (como, por exemplo, recuperar as intervenções) foram bem sucedidas, apesar de 
apresentar lentidão. Todavia, requisições com uma carga maior de dados (como o cadastro de uma colmeia) demoraram bastante tempo, fato que gerava atrasos no trabalho e, desse modo, comprometia a agilidade e eficiência proposta pelo sistema.

Entretanto, nos experimentos realizados em um ambiente com acesso a rede de área local sem fio, os resultados se mostraram satisfatórios. As aplicações funcionaram como previsto na maioria dos casos e foi possível realizar o trabalho de gerenciamento de apiários, colmeias e colônias de modo convincente. Nesse sentido, os formulários sugeridos para as visitas a um apiário ou colmeia se caracterizaram como adequados, sendo capazes de manter os mais relevantes conhecimentos acerca das questões.

\section{Proposta de demonstração}

A demonstração visa apresentar as funcionalidades tanto do módulo web quanto do aplicativo móvel, e consiste na utilização de dois computadores, um celular e um tablet. Um desses computadores conterá o banco de dados, o servidor de aplicação com o BeeCheck web e a API, além de um navegador acessando o BeeCheck web, que focará na demonstração do cadastro dos dados, como pode ser visto no computador 1. Já o computador 2 conterá um navegador acessando o BeeCheck web e focará na demonstração da visualização dos dados cadastrados pelo computador 1 e pelo BeeCheck mobile. $\mathrm{O}$ celular indicado no item 3 e o tablet indicado no item 4 farão a demonstração de intervenções e manejos de apiários feitos em dois apiários diferentes, além de demonstrar o uso do aplicativo móvel em diferentes dispositivos. Um esquema da demonstração pode ser encontrado na Figura 4.

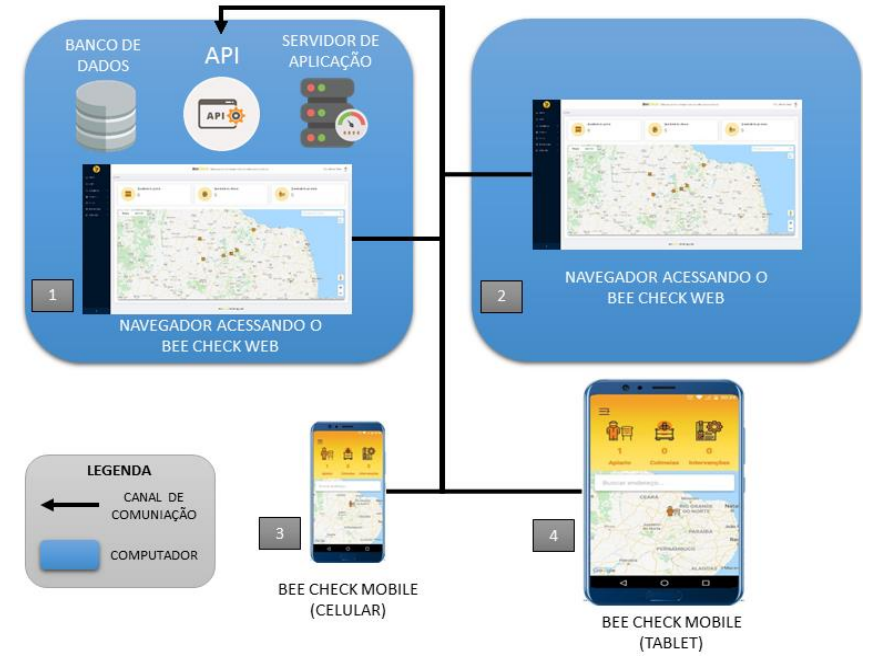

Figura 4. Esquema da proposta de demonstração

\section{Considerações Finais}

Em suma, realizar o armazenamento de informações dos apiários e/ou colmeias em papéis constitui um processo enfadonho e inseguro. Esses dados podem ser facilmente violados caso o papel seja danificado ou perdido. Dessa forma, a plataforma BeeCheck torna-se bastante útil na Escrituração Zootécnica, uma vez que as informações ficam armazenadas em uma base de dados confiável, tornando a perca dos registros improvável.

De acordo com os resultados obtidos durante os experimentos, pode-se constatar que o aplicativo móvel cumpriu parcialmente os objetivos, com pequenos problemas que 
serão solucionados em uma nova versão do projeto. Dentre essas melhorias, diante da má qualidade da conectividade no campo, pretende-se implementar o recurso offline first, que permite o uso do aplicativo de forma offline, sincronizando suas informações com os dados em nuvem assim que o dispositivo for conectado com a internet. Além disso, é almejada a implantação de uma rica base de dados através do procedimento de aquisição do conhecimento contínuo. Espera-se, por fim, que a contribuição advinda deste projeto venha a somar positivamente na área da apicultura, tornando o trabalho mais produtivo.

\section{URL's}

- Documentação: https://bee-check-ifrn.gitbook.io/;

- Manual, download e tutorial de instalação do aplicativo: https://drive.google.com/drive/folders/1FjNiS_C4BQs9oKM4To8 FFtkF0W y0 y?usp=sharing;

- Vídeo do sistema web: https://www.youtube.com/watch?v=jBc60NJ0DxA;

- Vídeo do aplicativo: https://www.youtube.com/watch?v=O6Hg2ZIvhpI;

- URL do sistema web: http://bee-check.herokuapp.com;

- Repositório do aplicativo: https://github.com/victor-kayan/BeeCheck-mobile;

- Repositório do sistema web: https://github.com/victor-kayan/BeeCheck-web;

- Repositório da API: https://github.com/victor-kayan/BeeCheck-api;

- Credenciais para acesso: e-mail do aplicativo: claudio@gmail.com, e-mail para o website: abreu@gmail.com, senha para ambos: 123456.

\section{Referências}

Associação Brasileira de Estudos das Abelhas (A.B.E.L.H.A). Apicultura no Brasil. Site institucional. Disponível em $<$ https://abelha.org.br/apicultura-no-brasil/>. Acesso em: 16 de apr. 2019.

Bacaxixi, P. et al. A importância da apicultura no Brasil. Revista Científica Eletrônica de Agronomia , v. 10, n. 20, 2011.

Couloris, G.; Dollimore, J. e Kindberg, T. 2007. Distributed Systems: Concepts and Design. Addison-Wesley.

Dutra, T. F. S. Beehiveior - Sistema de monitoramento e controle de colmeias de produção apícola. 2016. Dissertação de Mestrado. Brasil.

Iordache, B. Caderno do apicultor. v. 4. 22 Mar. 2019. Disponível em $<$ https://play.google.com/store/apps/details?id=com.csg.apiarybook $>$. Acesso em: 07 mai. 2019.

Lôbo, R. N. B. Importância da Escrituração Zootécnica para o Desenvolvimento da Caprino-ovinocultura. $2007.20 \mathrm{em}$ : $<$ http://srvgen.cnpc.embrapa.br/pagina/escrit.php>. Acesso em: 24 nov. 2019.

Oliveira, F. M. Fundamentals of Brazilian Honey Analysis: An Overview. In: Toledo, V. A. A. (Ed.). Honey Analysis. Rijeka: IntechOpen, 2017. p. 139 - 170.

Rodrigues, L. C. R. Arquitetura REST. 2009. Monografia (Bacharelado em Ciência da Computação) - Instituto de Ciências Exatas da Universidade Federal de Juiz de Fora, Juiz de Fora, 2009.

Silva, R. A. da, et al. Composição e propriedades terapêuticas do mel de abelha. Alimentos e Nutrição Araraquara, v. 17, n. 1, p. 113-120, 2008.

Souza, D. C. Apicultura: manual do agente de desenvolvimento rural. 2a Edição. Brasília: SEBRAE, 2008. 